\title{
A Comparative Study of Two Reference Estimation Methods in EEG Recording ${ }^{\star}$
}

\author{
Sanqing $\mathrm{Hu}^{1, \star \star}$, Yu $\mathrm{Cao}^{2}$, Shihui Chen ${ }^{1}$, Jianhai Zhang ${ }^{1}$, Wanzeng Kong ${ }^{1}$, \\ Kun Yang ${ }^{1}$, Xun $\mathrm{Li}^{1}$, and Yanbin Zhang ${ }^{1}$ \\ 1 College of Computer Science \\ Hangzhou Dianzi University, Hangzhou, Zhejiang, China \\ ${ }^{2}$ College of Engineering and Computer Science \\ The University of Tennessee at Chattanooga \\ Chattanooga, TN 37403 USA \\ \{sqhu, jhzhang, kongwanzeng, yangkun, \\ zhyb, xunli\} @hdu.edu.cn, \\ yu-cao@utc.edu
}

\begin{abstract}
In [1] we proposed two methods to identify the reference electrode signal under the key assumption that the reference signal is independent from EEG sources. This assumption is shown to be possibly true for intracranial EEG with a scalp reference. In this paper, we theoretically prove that the obtained reference signal by using the second method in [1] or the equivalent MPDR approach [2] outperforms the widely used average reference (AR) if the real reference is independent from EEG sources. The simulation results confirm the advantages over AR.
\end{abstract}

Keywords: EEG, Reference signal, Independent component analysis, Average reference.

\section{Introduction}

The unsurpassed temporal resolution of electroencephalography (EEG) has led to its widespread use by clinicians and scientists investigating physiologic and pathologic brain function. The EEG signal reflects the difference between electrical potentials measured at two different electrodes, and as such it is always necessary to select a reference electrode. The vast majority of clinical and research EEG recordings, both scalp and intracranial, are obtained using a common cephalic reference. As a result, the reference signal generally has an effect on the EEG. To reduce this effect, a neutral potential is the most desirable reference. However, it is impossible to find a point of neutral or zero

\footnotetext{
* The work was partially supported by National Natural Science Foundation of China under Grants (61070127, 61102028, 61100102), International Cooperation Project of Zhejiang Province, China (No. 2009C14013), Qianjiang Project of Zhejiang Province, China, under Grant 2011R10063, U.S.National Science Foundation (NSF) under Grant 0821820, and Tennessee Higher Education Commission, the State of Tennessee, USA, and NIH R01 NS063039.

${ }^{\star \star}$ Corresponding author.
} 
potential on the body surface [3]. Physically, the potential at infinity is the ideal reference since it is far from all the neural sources, and would have no effect on the EEG recordings. However, such a distant reference is not realistic in practice [4]. Therefore, the widely used cephalic electrode, non-cephalic electrode, earlobe, neck, and average references etc. each generally have an effect on both scalp and intracranial EEG recordings. In fact, the effect of an active reference on EEG recording is one of the oldest technical problems in the study of human EEG [45].

Reference signal, especially reference signal contaminated by non-cerebral artifacts like muscle, EKG etc., not only hinders visual interpretation of the EEG tracings, but also complicates quantitative analysis of EEG (see [6] and references therein). Due to important impact of reference signal, most of researchers in EEG field focus on average reference EEG which is reference-free EEG. Here the primary difficulty is that we do not know apriori how many cephalic electrodes are adequate to get an unbiased AR. There is clear evidence that the 10-20 system coverage is inadequate [7 [8] and a full dense coverage of the head surface is necessary for a good average scalp EEG reference [9]. Additionally, calculating a common AR from a selection of implanted grids, strips, and depth electrodes is not well supported geometrically [5].

Recently independent component analysis (ICA) [10] has received wide attention in biomedical engineering [11]-[15]. Especially in [1] we applied ICA technology to estimate reference signal from intracranial EEG for the first time in the literature. In [1] based on the assumption of independence between scalp reference and intracranial sources we proposed two methods to obtain two reference signals called $\mathbf{R}_{1}$ and $\mathbf{R}_{2}$. $\mathbf{R}_{1}$ played an important role to explain why the obtained signal is close to the real reference signal. $\mathbf{R}_{2}$ was shown to be better than $\mathbf{R}_{1}$. The $\mathbf{R}_{2}$ method was shown to be equivalent to a constrained power minimization approach, that is, the minimum power distortionless response (MPDR) approach [2], which is the optimal solution in terms of least squared error as pointed in [2]. The above assumption of independence was verified by three patients for iEEG with scalp refernce [1]. Once this assumption is satisfied, the two methods in [1] can be applied to automatically identify the real reference and remove it from original referential EEG (scalp EEG or intracranial EEG).

In this paper, we theoretically prove that under the assumption of independence the obtained $\mathbf{R}_{2}$ signal is much better than the widely used AR. We showed that $\mathbf{R}_{2}$ is very close to the real reference $\mathbf{R}$ when the number of channels tends to be large enough, however, in general AR is not close to the real reference $\mathbf{R}$ even if the number of channels tends to infinity. Our simulation results confirm these theoretical results.

\section{Methods}

As described in [1], the EEG recordings can be described by the following multichannel model:

$$
\mathbf{X}(t)=\mathbf{R}(t) \mathbf{E}-\mathbf{B}(t) \quad \text { or } \quad \mathbf{X}(t)=\mathbf{R}(t) \mathbf{E}-\mathbf{A} \mathbf{S}(t)
$$


where $\mathbf{B}(t)=\left[b_{1}(t), \cdots, b_{n}(t)\right]^{T}$ and each $b_{i}$ is a localized region potential of the brain at the $i$-th surface electrode for scalp EEG or the $i$-th deep electrode for intracranial EEG (iEEG), $\mathbf{S}(t)=\left[s_{1}(t), \cdots, s_{m}(t)\right]^{T}$ and each $s_{i}$ is a source potential (or source for brevity) of the brain, $\mathbf{A}=\left[a_{i j}\right]$ is an $n \times m$ unknown constant mixing matrix determined by source locations and orientations, $\mathbf{R}(t)$ is a reference potential (or reference for brevity) from the common electrode (scalp electrode used in literature or body electrode used in this paper), $\mathbf{E}=[1, \cdots, 1]^{T}$ with $n$ unit $1, \mathbf{X}(t)=\left[x_{1}(t), \cdots, x_{n}(t)\right]^{T}$ and each $x_{i}$ is a measured potential difference between the common electrode and the $i$ th electrode, and $t=1, \cdots, N$ is sample time. In this model, we assume that each localized region potential $b_{i}$ is a linear mixture of $m$ source potentials $s_{1}, \cdots, s_{m}$ where each source potential $s_{i}$ actually consists of many local time-correlated dipole source potentials. This assumption is often made in the analysis of EEG signals. As such, Model (1) can be rewritten as

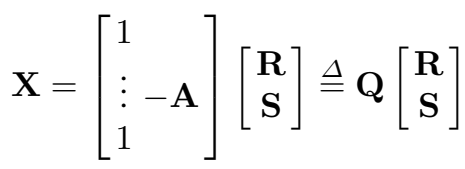

where $\mathbf{Q}$ is usually called the mixing matrix of dimension $n \times(m+1), \mathbf{X}$ is an $n \times N$ matrix, $\mathbf{S}$ is an $m \times N$ matrix, and $\mathbf{R}^{T}$ is a vector of dimension $N$.

In [1] we proposed two methods to obtain two reference signals called $\mathbf{R}_{1}$ and $\mathbf{R}_{2}$. $\mathbf{R}_{1}$ plays an important role to explain why the obtained signal is close to the real reference signal. $\mathbf{R}_{2}$ is shown to be better than $\mathbf{R}_{1}$.

The main idea in $\mathbf{R}_{2}$ is that the reference electrode signal is assumed to be independent from the scalp source electrode signals or intracranial source electrode signals. The independence between scalp reference electrode signal and intracranial electrode signals was verified by three patients in [1]. The method II in [1] can be described as follows: Let all independent components (ICs) of the bipolar montage EEG derived from (11) be $\bar{s}_{j}, j=1, \cdots, k$ where $k \leq m$. Then, $\mathbf{R}_{2}$ signal can be obtained as

$$
\mathbf{R}_{2}=\frac{1}{n} \sum_{i=1}^{n}\left[x_{i}-\sum_{l=1}^{k} \frac{E\left[x_{i} \bar{s}_{l}\right]}{E\left[\bar{s}_{l}^{2}\right]} \bar{s}_{l}\right]
$$

(see (7) in [1]).

\section{A Comparative Study: $R_{2}$ Signal and Average Reference}

It is noted that in the first method [1] ICA is applied to obtain all independent components having variance of 1 and mean value of zero [16]. In this section, without a loss of generality for equation (1) we always assume that $\mathbf{R}$ and all sources $s_{1}, \cdots, s_{m}$ are independent and each has variance of 1 and mean value of zero. Next we will theoretically prove that $\mathbf{R}_{2}$ signal is much better than AR by calculating correlation coefficients between $\mathbf{R}$ and $\mathbf{R}_{2}$, and between $\mathbf{R}$ and $A R$. Now we present our two main theoretical results as follows. 
Theorem 1: Assume that each $a_{i j}$ is independently uniformly distributed in the interval $[-a, a], i=1, \cdots, n, j=1, \cdots, m$. Then mean of correlation coefficient between $\mathbf{R}$ and $\mathrm{AR}$ (that is, $E[\operatorname{corr}(\mathbf{R}, \mathrm{AR})]$ ) is

$$
E[\operatorname{corr}(\mathbf{R}, \mathrm{AR})]=\frac{1}{\sqrt{1+\frac{m a^{2}}{3 n}}} .
$$

Proof. From (1) it follows that

$$
\mathrm{AR}=\frac{1}{n} \sum_{i=1}^{n} x_{i}=\frac{1}{n} \sum_{i=1}^{n}\left(\mathbf{R}-\left[a_{i 1}, \cdots, a_{i m}\right] \mathbf{S}\right)=\mathbf{R}-\frac{1}{n} \sum_{i=1}^{n} \sum_{j=1}^{m} a_{i j} s_{j}
$$

Since $\mathbf{R}$ is independent from all sources $s_{1}, \cdots, s_{m}$ and has variance of 1 , we have

$$
E[\mathbf{R} \times \mathrm{AR}]=E\left[\mathbf{R} \times\left(\mathbf{R}-\frac{1}{n} \sum_{i=1}^{n} \sum_{j=1}^{m} a_{i j} s_{j}\right)\right]=E\left[\mathbf{R}^{2}\right]=1 .
$$

Since $\mathbf{R}$ and all sources $s_{1}, \cdots, s_{m}$ are independent and each has variance of 1 and mean value of zero, we obtain the variance of $A R$ as

$$
\begin{aligned}
\sigma_{\mathrm{AR}}^{2} & =E\left[\mathrm{AR}^{2}\right]=E\left[\left(\mathbf{R}-\frac{1}{n} \sum_{i=1}^{n} \sum_{j=1}^{m} a_{i j} s_{j}\right)^{2}\right] \\
& =E\left[\mathbf{R}^{2}\right]-2 E\left[\mathbf{R} \times \frac{1}{n} \sum_{i=1}^{n} \sum_{j=1}^{m} a_{i j} s_{j}\right]+E\left[\left(\frac{1}{n} \sum_{i=1}^{n} \sum_{j=1}^{m} a_{i j} s_{j}\right)^{2}\right] \\
& =E\left[\mathbf{R}^{2}\right]+E\left[\left(\frac{1}{n} \sum_{i=1}^{n} \sum_{j=1}^{m} a_{i j} s_{j}\right)^{2}\right] \\
& =1+\frac{1}{n^{2}} E\left[\left(\sum_{i=1}^{n} \sum_{j=1}^{m} a_{i j} s_{j}\right)^{2}\right]=1+\frac{1}{n^{2}} E\left[\left(\sum_{j=1}^{m}\left(\sum_{i=1}^{n} a_{i j}\right) s_{j}\right)^{2}\right] \\
& =1+\frac{1}{n^{2}} \sum_{j=1}^{m}\left(\sum_{i=1}^{n} a_{i j}\right)^{2} E\left[s_{j}^{2}\right]=1+\frac{1}{n^{2}} \sum_{j=1}^{m}\left(\sum_{i=1}^{n} a_{i j}\right)^{2},
\end{aligned}
$$

so,

$$
\sigma_{\mathrm{AR}}^{2}=E\left[\mathrm{AR}^{2}\right]=1+\frac{1}{n^{2}} \sum_{j=1}^{m}\left(\sum_{i=1}^{n} a_{i j}\right)^{2} .
$$

Since each $a_{i j},(i=1, \cdots, n, j=1, \cdots, m)$ is a random variable, from (7) we conclude that $\sigma_{\mathrm{AR}}^{2}$ or $E\left[\mathrm{AR}^{2}\right]$ is also a random variable. Based on the assumption that each 
$a_{i j}$ is independently uniformly distributed in the interval $[-a, a], i=1, \cdots, n, j=$ $1, \cdots, m$, from (7) we can obtain mean value of $\sigma_{\mathrm{AR}}^{2}$ or $E\left[\mathrm{AR}^{2}\right]$ as

$$
\begin{aligned}
E\left[\sigma_{\mathrm{AR}}^{2}\right] & =E\left[E\left[\mathrm{AR}^{2}\right]\right]=1+\frac{1}{n^{2}} \sum_{j=1}^{m} E\left[\left(\sum_{i=1}^{n} a_{i j}\right)^{2}\right] \\
& \left.=1+\frac{1}{n^{2}} \sum_{j=1}^{m} \sum_{i=1}^{n} E\left[a_{i j}^{2}\right] \quad \text { (based on independence among } a_{i j}\right) \\
& =1+\frac{1}{n^{2}} \times n \times m \times \frac{(a+a)^{2}}{12}=1+\frac{m a^{2}}{3 n} .
\end{aligned}
$$

From $\sigma_{\mathbf{R}}^{2}=1,(5)$ and $(8)$ it follows that

$$
\begin{aligned}
E[\operatorname{corr}(\mathbf{R}, \mathrm{AR})] & =E\left[\frac{E[\mathbf{R} \times \mathrm{AR}]}{\sigma_{\mathbf{R}} \times \sigma_{\mathrm{AR}}}\right]=E\left[\frac{1}{\sigma_{\mathrm{AR}}}\right]=\frac{1}{E\left[\sigma_{\mathrm{AR}}\right]} \\
& =\frac{1}{E\left[\sqrt{\sigma_{\mathrm{AR}}^{2}}\right]}=\frac{1}{\sqrt{E\left[\sigma_{\mathrm{AR}}^{2}\right]}}=\frac{1}{\sqrt{1+\frac{m a^{2}}{3 n}}} .
\end{aligned}
$$

Theorem 2: Assume that each $a_{i j}$ is independently uniformly distributed in the interval $[-a, a], i=1, \cdots, n, j=1, \cdots, m$. Then mean of correlation coefficient between $\mathbf{R}$ and $\mathbf{R}_{2}$ (that is, $E\left[\operatorname{corr}\left(\mathbf{R}, \mathbf{R}_{2}\right)\right]$ ) is

$$
E\left[\operatorname{corr}\left(\mathbf{R}, \mathbf{R}_{2}\right)\right]=\frac{1}{\sqrt{1+\frac{m a^{2}}{3 n}-\frac{k a^{2}}{3 n}}} .
$$

Its proof is omitted here for space reason.

From (4) and (9) we easily have the following corollary.

Corollary 1: i) Since $k \leq m, \forall m, n$, from (4) and (9) we can see

$$
E[\operatorname{corr}(\mathbf{R}, \mathrm{AR})]=\frac{1}{\sqrt{1+\frac{m a^{2}}{3 n}}}<\frac{1}{\sqrt{1+\frac{m a^{2}}{3 n}-\frac{k a^{2}}{3 n}}}=E\left[\operatorname{corr}\left(\mathbf{R}, \mathbf{R}_{2}\right)\right]
$$

which implies that $\mathbf{R}_{2}$ signal is always better than the average reference under the assumption of independence between the real reference and sources.

ii) When $m<n$, from (1) one can easily see $k=m$ in this case, then from Theorem 2 we can have

$$
E\left[\operatorname{corr}\left(\mathbf{R}, \mathbf{R}_{2}\right)\right]=1
$$

which means the obtained $\mathbf{R}_{2}$ signal is exactly the real reference $\mathbf{R}$. However, in this case, according to (4) average reference is not the real reference at all.

iii) For real EEG data, when $n$ is large enough, in general $m$ and $k$ are also large enough such that $\frac{m}{n} \approx 1$ and $\frac{m-k}{n} \approx 0$, then from (4) we get

$$
E[\operatorname{corr}(\mathbf{R}, \mathrm{AR})]=\frac{1}{\sqrt{1+\frac{m a^{2}}{3 n}}} \approx \frac{1}{\sqrt{1+\frac{a^{2}}{3}}} .
$$


From (9) we get

$$
E\left[\operatorname{corr}\left(\mathbf{R}, \mathbf{R}_{2}\right)\right]=\frac{1}{\sqrt{1+\frac{m a^{2}}{3 n}-\frac{k a^{2}}{3 n}}} \approx 1 .
$$

Therefore, even if $n$ (that is, the number of channels) tends to infinity, it is impossible for the obtained average reference to be close to the real reference $\mathbf{R}$. For example, independence between the scalp reference and intracranial sources was verified by three patients [1], hence, even if a large number of Grid electrodes are used for a patient, the obtained average reference from the recorded iEEG is not close to the real scalp reference at all. However, in this case the obtained $\mathbf{R}_{2}$ is very close to the real reference.

iv) From (4) and (9) one can see that both of $E\left[\operatorname{corr}\left(\mathbf{R}, \mathbf{R}_{2}\right)\right]$ and $E[\operatorname{corr}(\mathbf{R}, \mathrm{AR})]$ increasingly tend to 1 as $a$ decreasingly tends to zero, that means, the more large amplitude for the real reference, the more close to the real reference for $\mathbf{R}_{2}$ and average reference.

\section{Simulation Results}

In this section, we first present some simulation results to demonstrate the truth of our theoretical results. For real EEG data of $n$ channels, when fastICA is applied, we can generally get $n$ components. Accordingly we can get $n-1$ components for the bipolar montage EEG, so $k=n-1$. For the mixing matrix $\mathbf{A}$, three cases can be considered: overdetermined, square, and underdetermined. For simplicity, here we only consider underdetermined case for $\mathbf{A}$ (the other two cases can be similarly analyzed), e.g., $m=n+1$. In this case, from (4) we have

$$
E[\operatorname{corr}(\mathbf{R}, \mathrm{AR})]=\frac{1}{\sqrt{1+\frac{m a^{2}}{3 n}}}=\frac{1}{\sqrt{1+\frac{n+1}{n} \frac{a^{2}}{3}}}
$$

which monotonically increases to $\frac{1}{\sqrt{1+a^{2} / 3}}$ as $n$ tends to infinity. From (9) we get

$$
E\left[\operatorname{corr}\left(\mathbf{R}, \mathbf{R}_{2}\right)\right]=\frac{1}{\sqrt{1+\frac{m a^{2}}{3 n}-\frac{k a^{2}}{3 n}}}=\frac{1}{\sqrt{1+\frac{2 a^{2}}{3 n}}}
$$

which monotonically increases to 1 as $n$ tends to infinity. Let's consider (1) where $n=3,4, \cdots, 30$ and $m=n+1$, the real reference $\mathbf{R}$ is always the IC1 of Patient 3 [1], and the sources are from IC2, $\cdots$, IC32 of patient 3 [1]. For each $n$ we do 2000 realizations of $\mathbf{A}$ of $n \times(n+1)$ dimension whose entries are uniformly distributed in the interval $[-a, a]$. For all these 2000 realizations of $\mathbf{A}$ the sources are keep same, that is, when $\mathbf{A}$ is a $3 \times 4$ dimensional matrix, the source $\mathbf{S}=[\mathrm{IC} 2, \mathrm{IC} 3, \mathrm{IC} 4, \mathrm{IC} 5]^{T}$, when $\mathbf{A}$ is a $4 \times 5$ dimensional matrix, the source $\mathbf{S}=[\text { IC2,IC3,IC4,IC5,IC6 }]^{T}$, etc. For each realization we obtain $\mathbf{R}_{2}$ and AR and calculate correlation coefficient between $\mathbf{R}$ and AR (or $\mathbf{R}_{2}$ ), and then compute average value over these 2000 realizations, that is, $E[\operatorname{corr}(\mathbf{R}, \mathrm{AR})]$ or $E\left[\operatorname{corr}\left(\mathbf{R}, \mathbf{R}_{2}\right)\right]$. The results are shown in Fig. 1 1 where three cases for $a$ are considered: (A) $a=0.5$; (B) $a=1$; (C) $a=2$. From Fig. 1 lone can find that $\mathbf{i}$ ) $E\left[\operatorname{corr}\left(\mathbf{R}, \mathbf{R}_{2}\right)\right]>E[\operatorname{corr}(\mathbf{R}, \mathrm{AR})]$ for any one of three cases which is consistent with 
the conclusion in i) of Corollary 1. ii) Increasing trend holds for each curve (all curves related to $\mathbf{R}_{2}$ increase to $1, E[\operatorname{corr}(\mathbf{R}, \mathrm{AR})]$ in (A) increases to $0.96=\frac{1}{\sqrt{1+0.5^{2} / 3}}$, $E[\operatorname{corr}(\mathbf{R}, \mathrm{AR})]$ in (B) increases to $0.87=\frac{1}{\sqrt{1+1 / 3}}, E[\operatorname{corr}(\mathbf{R}, \mathrm{AR})]$ in $(\mathrm{C})$ increases to $\left.0.65=\frac{1}{\sqrt{1+2^{2} / 3}}\right)$, which are consistent with above theoretical analysis results. iii) The more small for $a$ (or the more large amplitude for the real reference signal $\mathbf{R}$ ), the more close to the real reference signal for the obtained $\mathbf{R}_{2}$ and AR. This conclusion was made in iv) of Corollary 1.
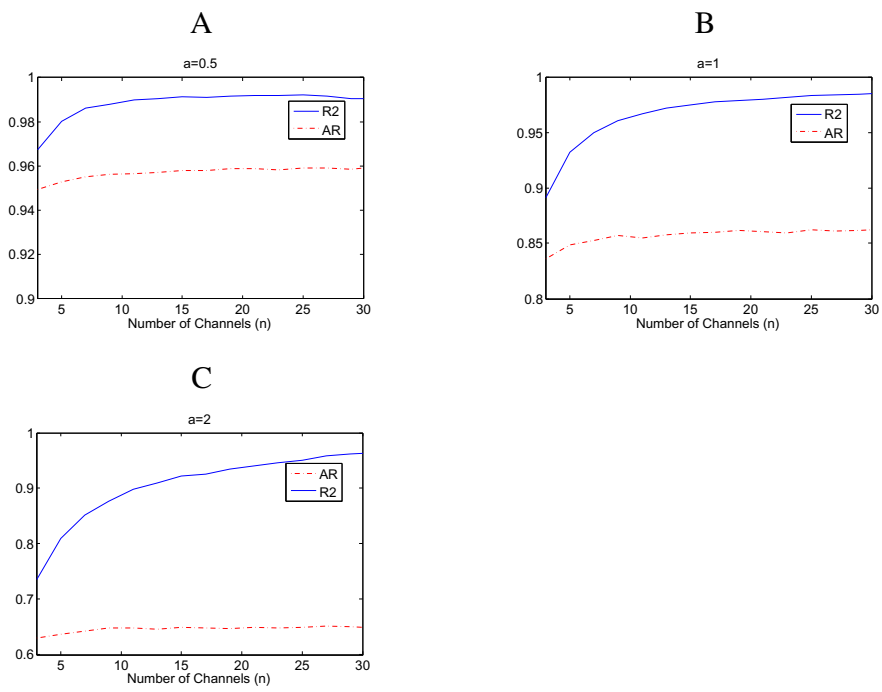

Fig. 1. Average value of correlation coefficients between $\mathbf{R}$ and $\mathrm{AR}$ (or $\mathbf{R}_{2}$ ) for 2000 realizations of $\mathbf{A}$ when the number of channels $n$ is given $(n=3,4 \cdots, 29,30)$. (A) $a=0.5$. (B) $a=1$. (C) $a=2$.

\section{Discussion and Conclusions}

In [1] we proposed a novel method ( $\mathbf{R}_{2}$ method) to estimate the real reference signal in EEG recording. The key issue in this method requires that the reference electrode signal is independent from all source signals. In [1] the independence between the scalp reference and intracranial sources was verified by three patients. Hence, $\mathbf{R}_{2}$ signal may provide a better way to estimate the real reference only with a simple assumption of independence. It is noted that this requirement is different from the assumption [17] that the reference signal is a linear mixture of scalp sources. As such, the method in [17] cannot be used in the case that the reference signal is independent from sources, e.g., intracranial EEG.

Another widely used reference in EEG field is AR. In this paper, we theoretically proved that under the assumption of independence the obtained $\mathbf{R}_{2}$ signal is better than AR. We showed that $\mathbf{R}_{2}$ is very close to the real reference $\mathbf{R}$ when the number 
of channels tends to be large enough, however, in general AR never closes the real reference $\mathbf{R}$ even if the number of channels tends to infinity. Our simulation results were consistent with these theoretical results.

\section{References}

1. Hu, S., Stead, S., Worrell, G.: Automatic identification and removal of scalp reference signal for intracranial EEGs based on independent component analysis. IEEE Trans. on Biomedical Engineering. 54, 1560-1572 (2007)

2. Ranta, R., Madhu, N.: Reference Estimation in EEG: Analysis of Equivalent Approaches. IEEE Signal Processing Letters 19, 12-15 (2012)

3. Geselowitz, D.B.: The zero of potential. IEEE Eng. Med. Biol. Mag. 17, 128-132 (1998)

4. Nunez, P.L.: Electric fields of the brain: the neurophysics of EEG. Oxford University Press, New York (1981)

5. Schiff, S.J.: Dangerous Phase. Neuroinformatics 3, 315-318 (2006)

6. Hu, S., Stead, S., Dai, Q., Worrell, G.: On the recording reference contribution to EEG correlation, phase synchrony and coherence. IEEE Transactions on SMC-Part B 40, 1294-1304 (2010)

7. Guevara, R., Velazquez, J.L., Nenadovic, V., Wennberg, R., Senjanovic, G., Dominguez, L.G.: Phase synchronization measurements using electroencephalographic recordings: what can we really say about neuronal synchrony? Neuroinformatics 3, 301-314 (2005)

8. Bertrand, O., Perrin, F., Pernier, J.: A theoretical justification of the average reference in topographic evoked potential studies. Electroencephalogy Clin. Neurophysiol. 62, 462-464 (1985)

9. Junghofer, M., Elbert, T., Tucker, D.M., Braun, C.: The polar average reference effect: a bias in estimating the head surface integral in EEG recording. Clin. Neurophysiol. 110, 11491155 (1999)

10. Hyvarinen, A., Oja, E.: Independent component analysis: algorithms and applications. Neural Networks 13, 411-430 (2000)

11. Senhadji, L., Kachenoura, A., Albera, L., Comon, P.: On the use of independent component analysis techniques in the field of brain computer interface. IRBM 30, 211-217 (2009)

12. Kachenoura, A., Albera, L., Senhadji, L., Comon, P.: ICA: a potential tool for BCI systems. IEEE Signal Processing Magazine, Special Issue on Brain-Computer Interfaces 25, 57-68 (2008)

13. Albera, L., Comon, P., Parra, L., Karfoul, A., Kachenoura, A., Senhadji, L.: Biomedical applications. In: Comom, P., Jutten, C. (eds.) Handbook of Blind Source Separation. Academic Press (2010)

14. Onton, J.L., Westerfieldb, M., Townsendb, J., Makeiga, S.: Imaging human EEG dynamics using independent component analysis. Neuroscience \& Biobehavioral Reviews 30, 808-822 (2006); J. Neurosci Methods 134, 9-21 (2004)

15. Urrestarazu, E., LeVan, P., Gotman, J.: Independent component analysis identifies ictal bitemporal activity in intracranial recordings at the time of unilateral discharges. Clin. Neurophysiol. 117, 549-561 (2006)

16. http://www.cis.hut.fi/projects/ica/fastica/

17. Yao, D.: A method to standardize a reference of scalp EEG recordings to a point at infinity. Physiol. Meas. 22, 693-711 (2001) 